\title{
Vertical Facial Midline Variations in Ethnic Groups
}

\author{
Ashwini Bhangale ${ }^{1}$, Ajay Kakar ${ }^{2}$, Maria Csillag ${ }^{3 *}$ \\ ${ }^{1}$ Working Clinician for the Study, Mumbai, India \\ ${ }^{2}$ Periodontist, Mumbai, India \\ ${ }^{3}$ Principal Author and Creator of the Smylist ${ }^{\circledR}$ Concept, Aesthetic Dentist, Budapest, Hungary, Europe \\ *Corresponding Author: Maria Csillag, Principal Author and Creator of the Smylist ${ }^{\circledR}$ Concept, Aesthetic \\ Dentist, Budapest, Hungary, Europe; Email: maria.csillag@smylist.com
}

Received Date: 05-10-2020; Accepted Date: 23-10-2020; Published Date: 31-10-2020

Copyright $^{\Theta} 2020$ by Bhangale A, et al. All rights reserved. This is an open access article distributed under the terms of the Creative Commons Attribution License, which permits unrestricted use, distribution, and reproduction in any medium, provided the original author and source are credited.

\begin{abstract}
This study was carried out to evaluate the incidence and pattern of facial midlines across three ethnic grtoupsd of populations. The ethnic groups being studied are Caucasian, African and Oriental. The Smylist philosophy has introduced the concept of different kinds of vertical facial midlines. Based on this concept the distribution and incidence of straight, curved and sloped midlines, as propounded by Smylist, was assessed. This study can be considered as an extension or follow up of a study done on an Indian population to assess the incidence of facial midlines which was published in 2019. There were 50 subjects included in each of the ethnic groups to make a total of 150 subjects. A photo documentation was used for each of the subjects. The pictures were standardized in the straight face Cheese A picture as defined in the Smylist concept. An attempt was made to keep an equal number of male and female subjects. All the pictures were loaded on the Smylist software and the facial analysis was carried out to generate and identify the type of midline present. The results showed that the incidence of the straight midline was the lowest in all the three ethnic groups. The curved and the sloped midline make up the bulk of the cases. The curved was the highest in the Caucasian group and the sloped was the largest percentage in the African and oriental groups. It can be safely concluded that the incidence of vertical straight midline is ostensibly less and should not be deliberated as a foundation for functional and aesthetic rehabilitation. This is consistently observed in all kinds of ethnic groups and follows the pattern of the Indian study. It can also be concluded that the curved and sloped incidences vary considerably in different ethnic groups.
\end{abstract}

Bhangale A | Volume 1; Issue 3 (2020) | JDHOR-1(3)-014 | Research Article

Citation: Csillag M, et al. Vertical Facial Midline Variations in Ethnic Groups. J Dental Health Oral

Res. 2020;1(3):1-9.

DOI: http://dx.doi.org/10.46889/JDHOR.2020.1303 


\section{Keywords}

Converging; Facial Midline; Facial symmetry; Smylist; Straight; Sloped; African; Caucasian; Oriental; Ethnic

\section{Introduction}

Humans have evolved significantly over the centuries, with the face being the most evolved trait in the entire body. The ratio and proportion of the face has also altered in this evolutionary pattern. Aesthetic dentistry is an amalgamation of art and science and the dental profession has usually addressed the aesthetic component as a standalone consideration. One very important factor that should be considered in this process is the functional aspect. The two should be blended together to ensure a very ideal end result with good longevity. Over years these two aspects have been dealt with individually without considering any interplay between them. Even the aesthetic component, when studied, assumes a lot of aspects. These assumptions may not be true. The Smylist concept is the first such concept which has propounded the theory that the human face is not necessarily symmetrical in terms of size and shape when equally divided into two vertical halves. The Smylist concept goes on to establish the fact that ideal and proper functions is possible for the mandible and consequently for the entire body only if the asymmetry of the face is taken into consideration. Determining the accurate facial structure and its symmetries can be carried out accurately only with special tools with great ease and comfort.

The advent of photography and now digital photography is the start point of studying the structure of the face. Using the photographs of individual it becomes possible to assess the structure and symmetry of the face [1]. Various planes, lines and the reference points have been reported and used to establish he vertical midline of the face [2]. This midline is used while planning or implementing surgically, orthodontic, prosthetic or restorative procedures [3-5]. While carrying out the analysis of the face horizontal and vertical lines are plotted by using the reference points. The Smylist Aesthetic software is an invaluable tool in assessing the horizontal lines as well as the vertical facial midline.

\section{Review of Literature}

A look into the literature shows that the vertical midline is established using the nasion, the tip of the nose and the philtrum. Conventionally the pogonion point on the chin is also used to complete the vertical facial midline [6-8]. The incisive papilla is considered as a midline intra orally. It is agreed upon that, the facial midline should be established during rehabilitation and that the midline should be in close approximation with the mesial surfaces of the maxillary incisors. It has also been suggested by Latta that the maxillary midline should be coinciding 
the facial midline and if not should be at least parallel to the same [9]. In addition horizontal lines on the face are also considered. These are plotted on the face from the left to the right eyebrow, the interpupillary line, inter nasal line and the inter commissure line.

A deviation of not more than $4 \mathrm{~mm}$ of the maxillary midline from the facial midline is not as noticeable by the patients or the dentist as suggested in a study [10]. A symmetric face is the one which has equilibrium concerning both the hard and soft tissues. An aesthetically attractive face not only has precisely aligned planes and segments but also suitable analogous facial midline. Several studies have been carried out which confirm that the face is asymmetrical [11]. Facial and dental parameters and proportions fluctuate amid the diverse ethnic groups which seemingly are a consequence of distinction in the anatomy of the fundamental hard and soft tissues [12,13]. Some studies suggest that a hypothetical midline can be assumed from the mid of the upper lip or the philtrum [5]. Yet, a common notion has been followed that the facial midline is straight and the further restoration is based on this assumption. An independent study carried out in the previous years has remarkably revealed that the reference points evidently didn't accord with the straight vertical midline [14].

The Smylist concept utilizes the standardized geometric guidelines to overcome the setbacks confronted owing to an asymmetric face. The pogonion is not considered as a reference point since the mandible may be rotated to the left or the right and could actually lead to an incorrect vertical facial midline. The desirable pogonion point can be digitally determined after establishing the vertical facial midline by using the Smylist landmarks and horizontal lines. The midline may be straight but can be often curved or sloped. The curved or sloped midlines can be either skewed to right or left side. Establishment of the facial midline is the stepping stone for aesthetic and functional dental rehabilitation. In reality, inter-incisal and the occlusal planes are extremely dependent on an accurate vertical facial midline. This has hardly been spoken about in the literature. In a recent study carried out on 100 subjects from India, the facial midline pattern astonishingly revealed that only $5 \%$ of them had straight facial midline, whereas $68 \%$ and $27 \%$ had sloped and curved facial midlines, respectively. The foremost purpose of this study is to conclude prevalence of facial straight, sloped and curved midlines in adult human populations across three ethnic groups, namely Caucasian, African and the Oriental.

\section{Materials and Method}

A total of 150 subjects were included in this study. The age range was from 25-70. An equal distribution between males and females was attempted. There were a total of 78 females and 72 males. There were 50 candidates in each ethnic group. A consent was obtained for 
publication of the picture of those subjects whose pictures appear in the study. The inclusion and exclusion criteria were

- Should be between the age of 30 and 60

- Should not have any history of intraoral trauma

- Should not have advanced active periodontal disease

- Should not be undergoing orthodontic treatment

- Should not have more than 4 missing teeth

- Should not be having Downs syndrome

The photographs used were of the front face with the patient smiling in what is termed as the Cheese A picture in the Smylist concept were taken in a straight profile and with 'Cheese A' format which evidently portrayed both the anterior dentition. The pictures revealed clearly visible maxillary dentition. All the pictures were in the digital format. The pictures were then loaded on the Smylist Aesthetic Software ${ }^{\circledR}$. The software was used to plot the four horizontal lines and the vertical facial midline for all the photographs. The horizontal lines were are as follows:

1. From the right to the left eyebrow

2. The Inter-pupillary line

3. The Inter-alar line

4. Inter-commissure line

After the horizontal lines were plotted, the vertical midline was plotted along the Glabella, tip of the nose and the philtrum. As recommended by the Smylist ${ }^{\circledR}$, pogonion was not included, since it is not a fixed position and depends on the rotation of the mandible to the left or right. The midline can then be extended to find out the desirable position of the pogonion. The horizontal lines can be either all parallel to the $\mathrm{X}$ axis, parallel to each other but canted to the left or the right, not parallel to each other and converging to the right or the left.

The convergence of the horizontal lines clearly demonstrate an asymmetry on the face and the vertical midline will always be curved in such a situation. This is an extremely important factor to consider during any future treatment planning. As mentioned earlier, the vertical facial midline is then drawn up. This midline can be either:

1. Straight

2. Sloped to the right or the left

3. Curved to the right or the left

The straight or sloped vertical facial midline can be associated with straight or canted horizontal patterns. When the horizontal lines converge to the left or right the vertical midline is always curved. The data was entered onto a spreadsheet and subjected to a statistical analysis. 


\section{Results}

The data was analysed and tabulated. There were a total of 150 subjects in the study. The gender distribution is presented in Table 1 .

\begin{tabular}{|c|c|c|}
\hline & Male & Female \\
\hline Caucasian & 24 & 26 \\
\hline African & 25 & 25 \\
\hline Oriental & 23 & 27 \\
\hline
\end{tabular}

Table 1: Gender distribution.

Table 2 depicts the pattern of the horizontal lines across all the subjects. If all the subjects across all the three groups is analysed, the scores of the three patterns straight, sloped and canted is $36 \%, 29.33 \%$ and $34.66 \%$. When this is compared to how it was distributed in the ethnic groups a very different pattern emerges. The Oriental group has a very high $48 \%$ of straight parallel horizontal lines while the Caucasian group is as low as $22 \%$. The African group is also on the higher side with $38 \%$. This is a very interesting finding and should be analyzed in more detail.

It follows that there will be more of the converging pattern seen in the Caucasian group which is as high as $56 \%$ and it is as low as $22 \%$ in the African group with the Oriental group being at 26\%. The Sloped parallel pattern shows the highest in Africans and lowest in the Caucasians with the Oriental group being $26 \%$.

\begin{tabular}{|c|c|c|c|c|c|c|c|}
\hline \multicolumn{4}{|c|}{ Parallel } & \multicolumn{3}{c|}{ Converging } \\
\hline & Straight & \multicolumn{3}{|c|}{ Canted } & \multicolumn{3}{c|}{ Canted } \\
\cline { 2 - 8 } & Total & Right & Left & Total & Right & Left & Total \\
\hline Caucasian & $22 \%$ & $0 \%$ & $22 \%$ & $22 \%$ & $36 \%$ & $20 \%$ & $56 \%$ \\
\hline African & $38 \%$ & $20 \%$ & $20 \%$ & $40 \%$ & $16 \%$ & $6 \%$ & $22 \%$ \\
\hline Oriental & $48 \%$ & $6 \%$ & $20 \%$ & $26 \%$ & $5 \%$ & $21 \%$ & $26 \%$ \\
\hline All groups & $36 \%$ & $8.60 \%$ & $20.60 \%$ & $29.33 \%$ & $18.66 \%$ & $16 \%$ & $34.66 \%$ \\
\hline
\end{tabular}

Table 2: Horizontal lines distribution.

Table 3 shows the pattern for the vertical facial midline across the three ethnic groups $6 \%$ of Caucasians had straight midline while the African and Oriental groups showed $8 \%$ straight midlines. The Caucasian group had 38\% sloped midlines, the African group $70 \%$ and the Oriental group had $66 \%$. The figures for the curved midline was $56 \%$ in Caucasians and $22 \%$ and $26 \%$ in the African and the Oriental group. 


\begin{tabular}{|c|c|c|c|}
\hline & Straight & Sloped & Curved \\
\hline Caucasian & $6 \%$ & $38 \%$ & $56 \%$ \\
\hline African & $8 \%$ & $70 \%$ & $22 \%$ \\
\hline Oriental & $8 \%$ & $66 \%$ & $26 \%$ \\
\hline All groups & $7.33 \%$ & $52.67 \%$ & $34.67 \%$ \\
\hline
\end{tabular}

Table 3: Vertical facial midline pattern.

A graphic depiction of the data is good for visualization and presented in the following graph (Fig. 1 and 2).

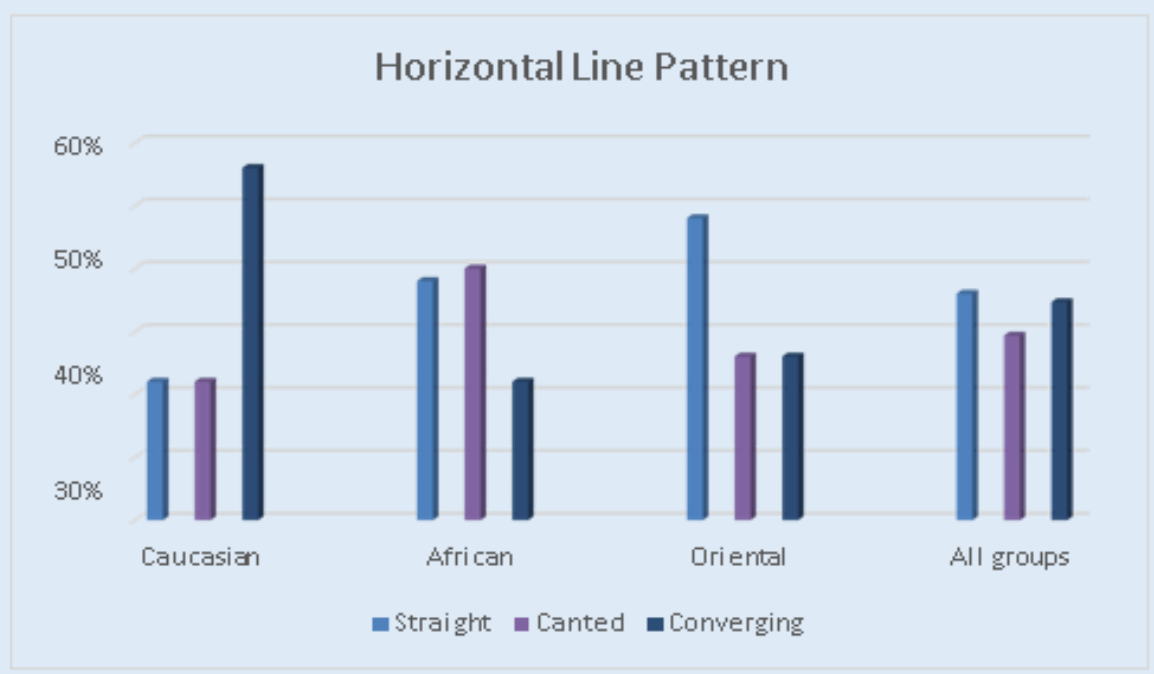

Figure 1: Horizontal lines distribution.

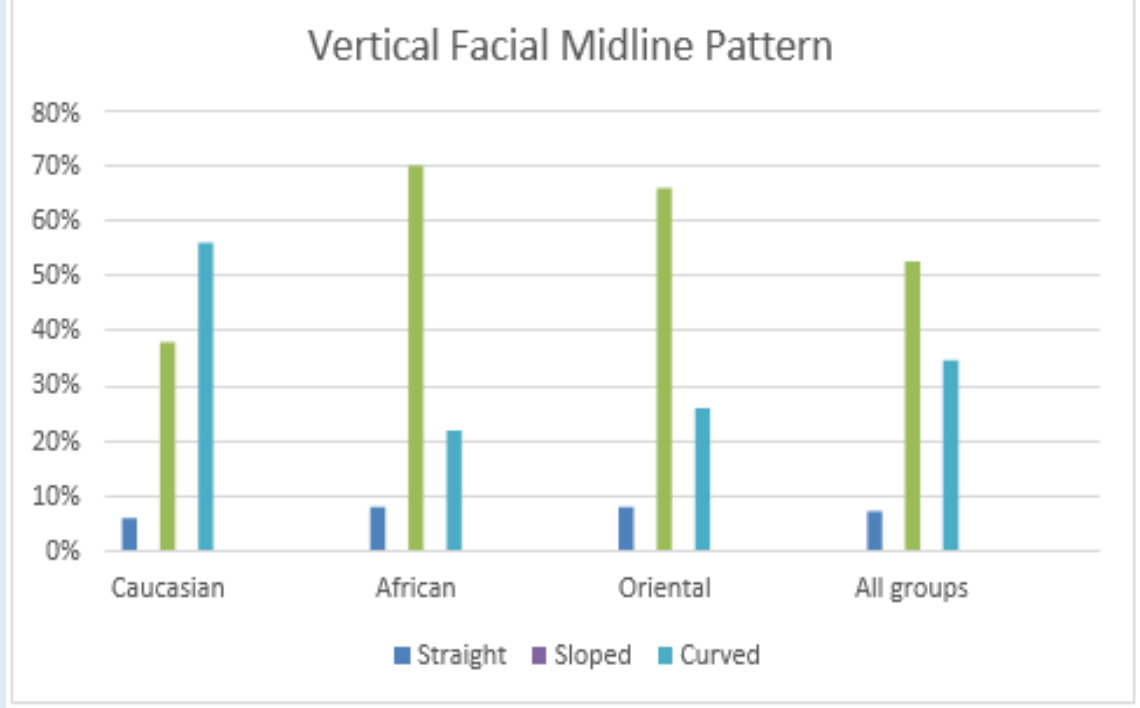

Figure 2: Pattern of vertical facial midlines. 


\section{Discussion}

In a previously published study by two of the authors of this publication, it was amply demonstrated that when variations from the normal are defined as acceptable variables, and dental rehabilitation done on the basis of the hypothetical straight midline always, leads to subsequent problems and frequent breakdown of the rehabilitated dentition [14]. The earlier study is substantiated with the evidence emerging from this study across three ethnic groups. In all the three groups the incidence of the straight midline is extremely low. Even though it was marginally higher than the Indian group of the previous study, it was still only mere 7.33 $\%$ across all the 150 subjects in this study. This pattern remained more or less consistent in all the three groups with a variation of only $1 \%$.

The sloped midline data presented some really very interesting information. The number of subjects in the three ethnic groups were the same and hence the groups can be compared statistically for variation in patterns. The overall percentage for sloped midlines was just beyond the half way mark at $52.67 \%$ but this was due to a large contribution from the African group. In this group $70 \%$ of the subjects were having sloped midlines while in the Caucasian group only $38 \%$ had a sloped midline. The Oriental group on the other hand had a percentage of $66 \%$ which is very close to the African group. The Caucasian group on the other hand clearly had a predominance of curved midlines which made up for $56 \%$ of its subjects. The African and the Oriental groups had only 22 and $26 \%$ subjects with curved midlines. This very wide range of variations in the midlines in the groups definitely indicates that there is a genetic predisposition towards either a curved or a sloped midline in ethnic groups which have different evolutionary patterns. The Indian data from the earlier study has been included in Graph 3 to show the similarities and differences in four ethnic groups (Fig. 3).

\section{Sloped and Curved Midlines}

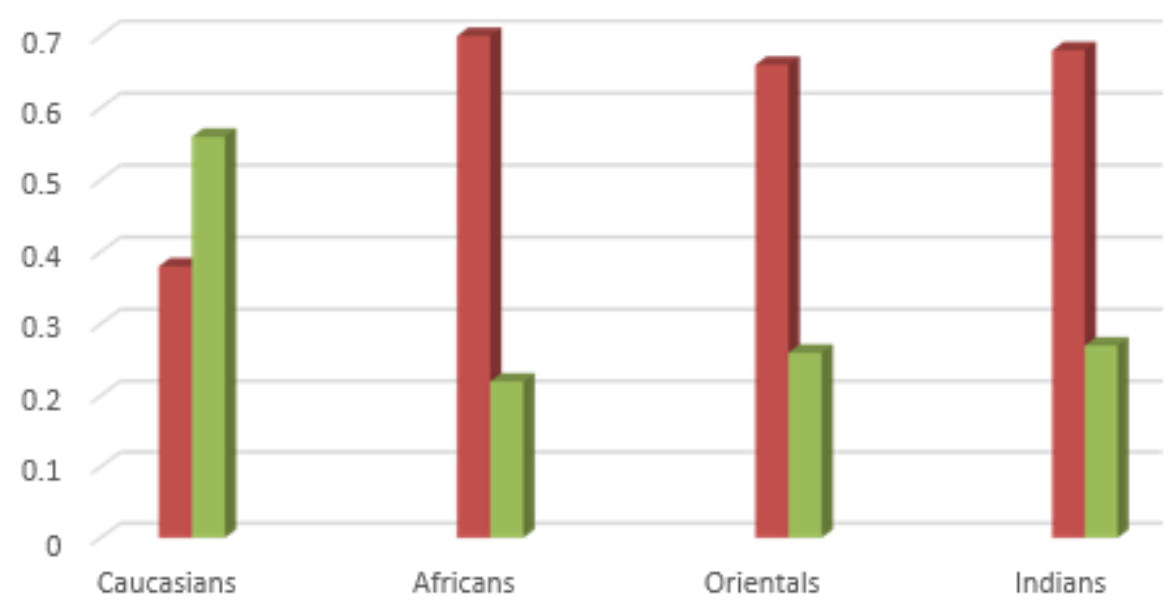

Figure 3: Comparing Sloped and Curved midlines across four ethnic groups. 
This study also establishes the fact that the straight vertical facial midline, generally accepted as the norm for all patients undertaking dental rehabilitative or aesthetic procedures, is not correct. It is an assumption which should not be followed. Rather every individual midline should be ascertained before embarking on any extensive dental therapy and the occlusal plane should follow the vertical facial midline of that particular individual. A similar finding was noted in the earlier study by the two authors [15]. This study opens the doors for further evaluation of sloped or curved midline subjects, wherein the degree of the slope or curve could be established and studied (Fig. 4).
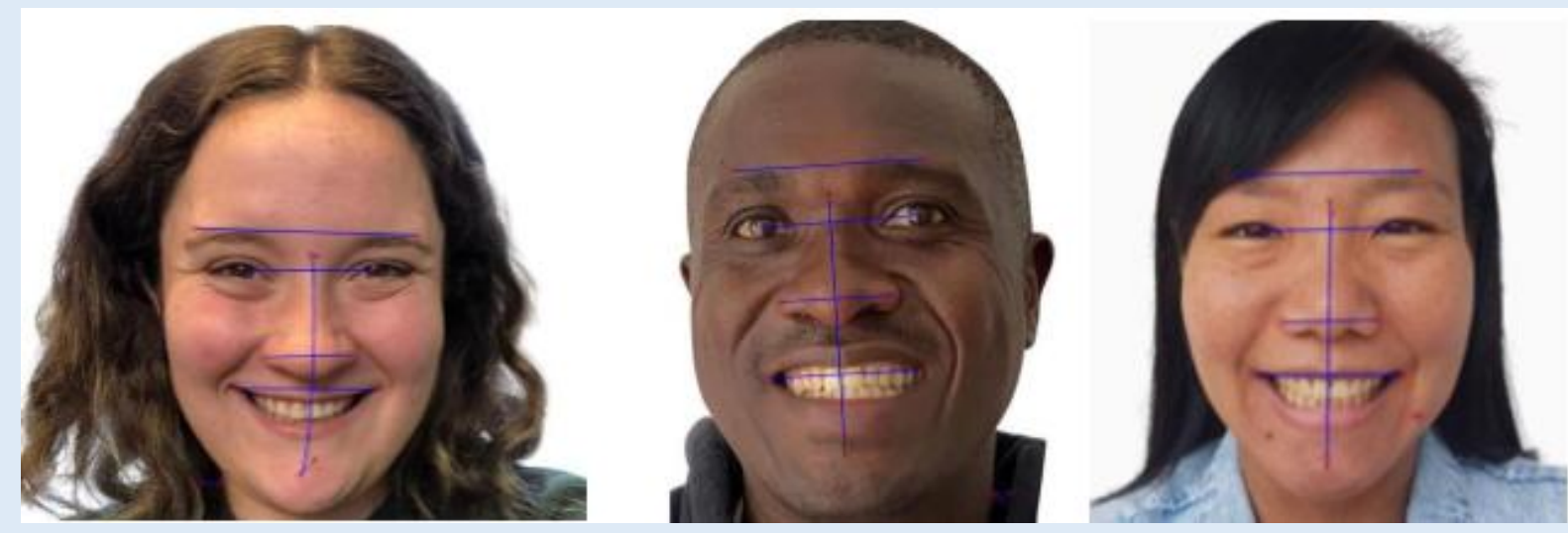

Figure 4: An example of a subjects from the all three ethnic groups.

Coupled with the data from the earlier study on Indian patients, most ethnic groups of human have been covered and patterns have emerged on what to expect. It is possible also in the future to study what percentage of individuals with sloped or curved midlines exhibit major dental discrepancies and damage which could be leading to systemic problems in the musculo skeletal system and other organs. These two studies are possible landmark studies in dental literature in the evolution of understanding the anatomy of the facial structures and its symmetry and its consequent effect on the dentition and the body.

\section{Conclusions}

The results of this study confirms the findings that the straight vertical facial midline is observed in very few individuals and is only seen in about $5-8 \%$ of the population. The sloped and the curved midlines account for the majority of individuals. The pattern of sloped and curved varies significantly between the Caucasian and the other two ethnic groups. The variation is very large in both the cases with the Caucasians having only $38 \%$ sloped midlines compared to the African group which has $70 \%$ sloped midlines. The reverse trend was noted in the Curved midlines. It can be concluded and stressed that each individual patient should be analysed for his/her facial symmetry and vertical facial midlines and dental aesthetics/rehabilitations should be based on the individual vertical facial midline. 


\section{References}

1. Duran GS, Dindaroğlu F, Görgülü S. Three-dimensional evaluation of social smile symmetry. Angle Orthod. 2017;87:96-103.

2. T Ray BR, Mathews VL. Artistic relationships in surface anatomy of the face: Application to reconstructive surgery. Plast Reconstr Surg. 1957;20(1):1-17.

3. González-Ulloa MA. Quantitative principles in cosmetic surgery of the face (profileplasty). Plast Reconstr Surg. 1962;29(2):186-98.

4. Peck H, Peck S. A concept of facial esthetics. Angle Orthod. 1970;40(4): 284-317.

5. Tjan AH, Miller GD, Josephene GP. Some esthetic factors in a smile. J Prosthet Dent. 1984;51(1):24-8.

6. Morley J, Eubank J. Macroesthetic elements of smile design. J Am Dent Assoc. 2001;132(1):39-45.

7. Bidra AS, Uribe F, Taylor TD, Agar JR, Rungruanganunt P, Neace WP. The relationship of facial anatomic landmarks with midlines of the face and mouth. J Prosthetic Dent. 2009;102(2):94-103.

8. Cardash HS, Ormanier Z, Laufer BZ. Observable deviation of the facial and anterior tooth midlines. J Prosthetic Dent. 2003;89(3):282-5.

9. Pound E. Lost-fine arts in the fallacy of the ridges. J Prosthetic Dent. 1954;4(1):6.

10. Kokich Jr VO, Asuman Kiyak H, Shapiro PA. Comparing the perception of dentists and lay people to altered dental esthetics. J Est Rest Dent. 1999;11(6):311-24.

11. Latta GH. The midline and its relation to anatomic landmarks in the edentulous patient. J Prosthetic Dent. 1988;59(6):681-3.

12. Miller EL, Bodden Jr WR, Jamison HC. A study of the relationship of the dental midline to the facial median line. J Prosthetic Dent. 1979 ;41(6):657-60.

13. McVay TJ, Latta Jr GH. Incidence of the maxillary midline diastema in adults. J Prosthetic Dent. 1984;52(6):809-11.

14. Bidra AS, Uribe F, Taylor TD, Agar JR, Rungruanganunt P, Neace WP. The relationship of facial anatomic landmarks with midlines of the face and mouth. J Prosthetic Dent. 2009;102(2):94-103.

15. Maria C, Ajay K. Incidence of straight, sloped and curved midlines in adult humans. Dent Res Oral Health. 2020;3(1):11-23. 